\title{
INVARIABLE Y PERTURBADOR, EL ENVEJECIMIENTO DEMOGRAFICO LANZA UN DESAFIO A LOS PODERES PUBLICOS
}

\section{INTRODUCCION}

La evolución demográfica, que incluye la transformación de la estructura de la población, cuando es juzgada como negativa o inquietante a largo plazo, puede inducir a los poderes públicos, en tanto que encarnación del Estado, a proponer ciertas medidas que se revelarán contraproducentes. Estas intervenciones pueden modificar, algunas veces, su evolución o, más bien, su dinámica. Las políticas de familia y las políticas sanitarias, son los ámbitos elegidos para quien quiere percibir y comprender la dialéctica población-gobierno, sin excluir otros ámbitos prioritarios como la educación o la vivienda.

\section{EL ENVEJECIMIENTO DE LA POBLACION, UN CONCEPTO ANTIGUO A REJUVENECER}

Cuando se evoca el envejecimiento de la población, normalmente basta con mencionar la definición del proceso, a saber, el aumento de la proporción de personas de edad avanzada, y a continuación se pasa la página. En otras pala-

Paul Paillat, demógrafo, gerontólogo, consejero científico de la Fundación Nacional de Gerontología (París). Antiguo jefe de Departamento del INED. 
bras, se pierde de vista lo esencial, es decir, la lenta y, por tanto, insidiosa transformación de la estructura de la población para todas las edades. El reemplazo de las generaciones se está produciendo continuamente, pero éstas cambian a un ritmo muy lento, hasta el punto de sorprender como si de una mutación profunda se tratase. Es un hecho que cualquier demógrafo es perfectamente capaz de medir y de predecir, y a los que valdría la pena escuchar. Surge, por tanto, la pregunta: ¿quién, de entre los que toman las decisiones en el mundo, se preocupa de las enseñanzas de la demografía?

El envejecimiento contemporáneo de las poblaciones europeas, incluida la nuestra, puede tener antecedentes, pero resulta difícil de imaginar modelos antiguos de envejecimiento con la versión actual que asocia una baja profunda y continua de la fecundidad a un alargamiento de la vida después de los sesenta años, asociación que no tiene como único resultado la acumulación de personas mayores.

La parte más importante de este proceso podría muy bien ser la transformación, sin precedentes, de los grupos centrales de la población (20-59 años), puesto que es de su vitalidad de lo que depende el dinamismo o, más concretamente, la supervivencia de una nación, o de un casi-continente como Europa.

A partir del momento en que los efectivos de jóvenes son demasiado débiles para tomar el relevo, debe esperarse una contracción numérica de la población que envejecerá de manera conjunta. En estos términos, es lícito inquietarse realmente, pero ¿es ésta la única actitud a adoptar? Ante esta despoblación no hay más respuesta que una vigorosa política pronatalista cuyo efecto, si se produce, como es de todos conocido, llevará decenios. A la espera de que el envejecimiento haya alcanzado a un nivel irreversible (aún por determinar), se corre el riesgo de la ineficacia. Por ejemplo, ¿no está ya Japón en vías de adoptar una política de inmigración de trabajadores, idea que tan sólo hace poco tiempo era herética y blasfema?

A nuestro entender, uno de los objetivos prioritarios de la investigación demográfica consistiría en medir los efectos directos de un crecimiento previsible del envejecimiento sobre el conjunto de la estructura de edades (y sexo) de la población. Teniendo en cuenta lo hecho hasta ahora, a nivel cuantitativo, el propósito puede parecer excesivo, pero examinando el aspecto cualitativo, se advierte rápidamente que no se ha hecho nada. Se trataría, por ejemplo, de rechazar la idea de que las personas (y los empleos) son intercambiables. Si esto no es así en el ámbito económico y si por esta razón se produce una resistencia a las medidas tomadas contra el desempleo, ¿por qué habrían de serlo en el ámbito social? Ni la constitución física, ni las capacidades intelectuales y psíquicas, ni el nivel de conocimientos son equivalentes de una persona a otra; a nivel colectivo, los grupos espontáneos son tan diferentes que hacen difícil el funcionamiento de la sociedad a la cual pertenecen.

La cuantificación de los efectivos sigue siendo una necesidad básica, puesto que permite el enmarque de los niveles de magnitud. Sólo el análisis cualitativo permite apreciar el potencial demográfico. Por ejemplo, la proporción de 
los analfabetos determinará las posibilidades de adaptación de un grupo, de una nación, a una modificación profunda de la tecnología; si esto no se tiene en cuenta, ¿este tipo de modificación se perderá? ¿Veremos en el futuro, más que en la actualidad, a algunos grupos de población abandonarse y deslizarse hacia el subproletariado?

A un nivel más modesto, más cercano a nuestras preocupaciones, el del cantón, por ejemplo, las deformaciones de la estructura demográfica provocadas por las emigraciones pasadas llegarán a obstaculizar el correcto funcionamiento de una política social allí donde falten las generaciones de cuarenta años, tan necesarias para los frágiles octogenarios.

Cuando numéricamente los detentadores y los defensores de las situaciones adquiridas sobrepasen a los candidatos en su reemplazo generacional, la sociedad correrá el riesgo de una tensión interna intergeneracional, o de un abatimiento de las nuevas generaciones. Este riesgo no es una certeza, pero cuando la pirámide se transforma en columna esta última obstruye el horizonte, a menos que se produzca una transformación en los conceptos heredados de una época en donde la población tenía otra estructura completamente diferente.

Nos proponemos explicitar lo que esta afirmación tiene de pertinente, tras la máscara de una suave provocación.

\section{FAMILIAS Y POLITICA FAMILIAR}

\section{Familias}

En las poblaciones europeas la familia constituye un núcleo elemental, aun cuando su estructura y duración hagan necesarias ciertas matizaciones de este aserto. En efecto, las familias de hecho (parejas no casadas con hijos jóvenes) ocupan un lugar creciente entre las personas menores de treinta años. Es todavía muy pronto para afirmar que esta evolución psicosociológica tenga consecuencias negativas sobre la fecundidad, pero ya se percibe que esta nueva estructura familiar puede influenciar la evolución demográfica en el futuro. Una población en la que el crecimiento disminuye o no subsiste más que gracias al retroceso de la mortalidad, no ofrece teóricamente un cuadro favorable a la constitución de familias numerosas. Pero, ¿cuántas parejas jóvenes se preguntan sobre su futuro, en este terreno, en función de lo que conocen acerca de la estructura demográfica actual y de su posible evolución? Su visión está, sin duda, más circunscrita a su horizonte personal y sobre todo a las perspectivas económicas, es decir, al empleo del hombre y, cada vez más, al de la mujer. A partir del momento en que la fecundidad sea cada vez menos espontánea, será menor la proporción de jóvenes en edad de casarse y de procrear, que van a convertirse en los factores-clave, fácilmente cuantificables, de la natalidad. En igual número, los efectivos de recién nacidos dependerán del comportamiento y la práctica de los padres potenciales, comportamiento en el cual entra 
en juego la imitación de modelos de referencia familiares o mediatizados, práctica que está ampliamente dominada por el conocimiento asimilado de los métodos contraceptivos o abortivos.

La pregunta a plantear es si ¿la fecundidad está o no en función de la existencia y de la disponibilidad de los abuelos? Si la respuesta es positiva, la multiplicación de éstos puede permitir esperar un relevo de la fecundidad. En el caso inverso, la ausencia de estos cuidadores familiares (y gratuitos) de niños puede frenar los proyectos de procreación. Dicho de otro modo, el aumento de la frecuencia de las familias de tres generaciones no puede ser considerada únicamente bajo un aspecto gerontológico. Asimismo, una mayor frecuencia de la cuarta generación puede, por el contrario, tener un efecto negativo. En efecto, los bisabuelos no pueden contar únicamente con sus hijos, que son sexagenarios; la carga suplementaria impuesta a sus nietos viene a competir con la de los biznietos. No se debe esperar que la joven madre de treinta y cinco años aumente su descendencia si se la obliga, al mismo tiempo, a ocuparse de su propia abuela (y/o de la de su marido). Es, sin embargo, alentador constatar que los «abuelos» actuales son más jóvenes, más libres y económicamente más independientes que sus antecesores. Todo iría a mejor si, paralelamente, no se hiciesen cargo de sus propios padres (en realidad, de su madre) cuya vida se alarga sin que tenga buena salud.

Nos falta experiencia real para evaluar las consecuencias de esta multiplicación de generaciones, dado que el fenómeno no está todavía muy difundido ${ }^{2}$, pero éstas requieren una observación continua que no proporciona el seguimiento de los censos. La aparición de los hijos en una estructura familiar estable o relativamente estable de forma piramidal, no se parece en nada a la situación que se presentará en el futuro cuando se generalicen las estructuras familiares poco estables y con generaciones múltiples, por no hablar de las estructuras de tipo "sueco» en las que a los abuelos «biológicos» se suman los abuelos «allegados» (en caso de nuevos matrimonios).

A esto hay que añadir que en las poblaciones verdaderamente envejecidas, como las nuestras en el futuro, las familias se alargarán, es decir, serán menos numerosas en cada generación, aunque el número de generaciones sucesivas coexistentes aumentará; aparece así, de nuevo, la imagen de la columna que hemos evocado para el conjunto de la población en la cual, ciertamente, las familias son las células elementales. A este respecto, la pregunta que se plantea es: ¿cómo podrá ser la política familiar la misma para las familias numerosas de tres generaciones que para las familias reducidas de cuatro (o cinco) generaciones?

2 En 1987, a los cincuenta y nueve años, un hombre de cada cuatro pertenecía a una familia de cuatro generaciones, según la encuesta longitudinal de la Fundación Nacional de Gerontología, Passages de la vie active à la retraite (París, PUF, 1989). 


\section{Politica familiar}

El objetivo natalista, ya expuesto, de la politica familiar llevado a cabo en Francia después de la Liberación, ha contribuido a reducir el concepto de familia al de la pareja con hijos jóvenes, disponiéndolo todo para que esas parejas se constituyeran y para que contribuyeran a la renovación de las generaciones, con un sistema impulsor (y no igualitario) de subsidios, acompañados de concesiones que, en algunos casos, han jugado un papel muy significativo en la reducción de la mortalidad materna e infantil ${ }^{3}$. Esta concentración de esfuerzos en torno a un objetivo determinado ha tenido, en mi opinión, una consecuencia nefasta; ha apartado de la familia a los ascendientes, ya escasamente mencionados en el Código Civil (con sólo siete menciones sobre los abuelos). ¡Los «viejos» no formaban parte de la familia que habían creado!

$\mathrm{Ni}$ que decir tiene que una nación que puede dotarse de una política familiar sin objetivo natalista, respondiendo, de este modo, a una preocupación de justicia social y de solidaridad. La incidencia directa sobre la evolución demográfica puede ser débil, pero no nula. ¿Qué dirección habría seguido la fecundidad sin este esfuerzo solidario?

El legislador no puede más que sentirse desamparado cuando se le hace observar la proporción creciente de las familias (biológicas) de cuatro generaciones coexistentes, es decir, incluyendo dos generaciones de jubilados (o más bien de jubiladas). Está forzado a admitir que la abuela es la hija de la bisabuela y también la madre de la joven esposa cuyos hijos son el objeto de atención del sistema de los subsidios familiares. Este se ve desconcertado cuando, con la ayuda de los accidentes de circulación, los abuelos (la segunda generación partiendo de la cúspide) se encuentran al cargo de los menores (cuarta generación), que pueden ser mañana los herederos directos de éstos (que, dicho sea de paso, tienen el derecho de adoptarles).

Otra consecuencia de esta decisión es la exclusión de la fratría, tan importante en ciertas culturas, aun cuando realmente los tíos y tías coetáneos de los padres jóvenes están mejor situados que los abuelos para tomar el relevo en caso de accidentes que, con tanta frecuencia, golpean a la generación intermedia (la tercera).

¿Cómo se pretende que la familia (en el sentido latino) sobreviva cuando sólo queda su esqueleto, si su estructura es únicamente descendente, olvidadiza del pasado que la ha prefigurado y en ocasiones condicionado? ¡Afortunadamente, el progreso de la genética médica nos recuerda que tenemos antecedentes!

Las leyes sucesorias, inspiradas bajo la preocupación de evitar la reconstrucción de las grandes fortunas de otras épocas, de dar preferencia al trabajo y

3 Debe recordarse que, durante la Liberación, Francia tenía la tasa de mortalidad infantil más elevada de Europa occidental (59 por 1.000), en tanto que actualmente es una de las más bajas $(4,4$ por 1.000$)$. 
no a la herencia como modo de adquisición y, en fin, de procurar recursos al Tesoro Público, contribuyen a limitar la expansión de las familias, en un momento en el que cada generación vive más tiempo que la precedente y podría desempeñar un papel más activo en comparación con las generaciones precedentes y siguientes. Con un promedio de vida que sobrepasará los ochenta años (de los cuales de diez a quince estupendos años transcurrirán a partir del inicio de la jubilación), ¿cómo admitir este desplazamiento del tejido social? ¿Cómo estar satisfecho con la imposición de obligaciones, sin proponer los medios para asumirlas?

De cara a una deformación sensible de la estructura de una población, bien sea en la base (como consecuencia de años de elevada fecundidad) o en la cúspide (como consecuencia de la acumulación de supervivientes de edad avanzada), se va a ejercer una presión sobre los poderes públicos con miras a obtener una protección social específica de los efectivos "excedentarios», es decir, de aquellos cuya dimensión excede, con mucho, el tamaño registrado anteriormente. Cuando una familia joven se amplía hasta el punto de que la vivienda anterior sea insuficiente, o de no permitir a la madre ejercer una actividad profesional, o cuando las parejas mayores constatan que se prolonga la longevidad de un abuelo, no necesariamente autónomo, del que se ocupan, unos y otros se vuelven hacia la colectividad para obtener ayuda y compensación a las cuales creen tener derecho. Estas demandas locales se dirigen, por diversos canales, a la cúspide (Parlamento, Gobierno); la respuesta se hace esperar y una vez dada ésta no satisface a nadie, en parte porque va por detrás de los acontecimientos, y en parte porque carece de todo carácter prospectivo.

Una respuesta de esta clase, aun cuando adecuada, se inscribe en un registro pasivo, bautizado abusivamente como "política». Animar a las parejas a tener hijos no es aceptable más que si, paralelamente, se arbitran disposiciones económicas y sociales encuadradas en un marco coherente, es decir, en una política merecedora de tal nombre. No es el caso de Francia, ya que, como hemos señalado, la visión pública de la familia es reduccionista. Animar más que por medio de las buenas palabras a los adultos a hacerse cargo de los padres muy ancianos que sufren una pérdida de autonomía, supondría que las necesidades propias de estas personas de cuarenta y cincuenta años fueran satisfechas, ya que el ámbito de las intervenciones no se puede limitar a la etapa que va más allá de los setenta o los ochenta años. Procurar techo y cuidados a las personas de más edad constituye un enorme progreso con respecto a la situación histórica, pero eso no es más que un paliativo. Procurar recursos es mucho más satisfactorio porque estos recursos, debidos y no concedidos, permitirán a sus titulares escoger, en lugar de contentarse con una ayuda de la que otros habrán diseñado la forma y fijado las modalidades.

El paso decisivo, en este campo, ha sido la generalización (por desgracia, heterogénea) de las pensiones de base y de las pensiones complementarias. En Francia, todos los asalariados no funcionarios pertenecen a uno (o varios) regímenes complementarios, de modo que el nivel de vida medio de un hogar de 
jubilados es bastante elevado, hasta el punto de suscitar alguna envidia entre los más jóvenes. Estos ingresos, emanados de los «derechos adquiridos», difieren singularmente de la ayuda aportada, bajo la condición de recurso, de los perceptores sociales cuyo número da la medida del retraso a cubrir.

Por otra parte, los subsidiados sociales están llamados a tener un mayor peso, serán más numerosos a la hora de percibir las prestaciones y serán más numerosos los que sobrevivan. Estadísticamente, los pobres no mueren tan rápidamente como antes. Dado que sus recursos son insuficientes, todo avance en este campo se traducirá en un agravamiento de la carga global, entorpecimiento que se reforzará por la llegada a la edad prefijada de generaciones más jóvenes, cuya mortalidad más débil cuando eran «adultos jóvenes» las habrá dejado a salvo. Tengamos en cuenta, además, el porvenir de las miríadas de parados de larga duración. A este respecto, el estado de salud de los futuros jubilados y sobre todo de los futuros subsidiados plantea un problema y demanda intervenciones preventivas dirigidas a mantener a las personas de cincuenta años (y a sus mayores) en forma. Cuanto más avanza la edad, más insuficientes y frágiles son las reparaciones. Cada vez los cuidados serán mayores pero no es seguro que se pueda curar por adelantado.

Cuando se parte de la familia biológica, según los grados (parentesco), superponiéndola la familia sociobiológica (los allegados), se permanece encerrado en un círculo restringido de intercambios que pueden ser muy ricos pero que, en nuestras sociedades occidentales, son frágiles, ya que están sometidos a tentaciones o presiones, e interferidos por las migraciones internas. La "política familiar", asociada con la "política de la vejez», debe tener en cuenta esta fragilidad, manteniendo los esfuerzos realizados en el seno de las familias, tanto en favor de los «jóvenes» como en favor de los «viejos». ¿Por qué no ayudar a los sexagenarios a ayudar, sin preocuparse de su edad?

$\mathrm{El}$ aporte de las migraciones externas puede resultar muy positivo en un momento de crisis histórica, tal como sucedió en Francia después de la primera guerra mundial. Estos inmigrantes son a menudo portadores de valores familiares de los que los franceses no poseen más que una vaga memoria, en la medida en que han conocido a sus abuelos (en el caso de las generaciones nacidas en los años veinte) o a sus bisabuelos (en las generaciones nacidas en los años cincuenta).

Cuando, como es habitual en nuestros días, estas familias no tienen raíces rurales, se entra en una fase de turbulencia y las autoridades descubren rápidamente que el apoyo que dichas familias podían esperar obtener de las redes familiares — que permanecen más vivas de lo que se cree generalmente- son aleatorias e intermitentes. Unicamente, «ayudando a los que ayudan» se superará la escasez de medios y de personal. Reconstruir los intercambios intrafamiliares (sin imponer reglas) es todavía una de las mejores formas para enriquecer los lazos entre los «ayudados» y los hijos que ayudan. Para tener éxito por esta vía es necesario reducir las distancias socioeconómicas que debilitan el equilibrio territorial. Cuando los mejores elementos, es decir, los más dinámicos, son tentados por los alicientes, verdaderos o míticos, de otra comunidad, 
toda la estructura demográfica de la comunidad de origen se deforma. ¿Dónde encontrar, entonces, las personas de cincuenta años, bien informadas y suficiente motivadas, para proporcionar ayuda a los frágiles «viejos»?

\section{La politica de la salud}

En una población envejecida y que continúa envejeciendo, la salud se convierte en el ámbito principal de la intervención pública. La sociedad no puede contentarse con gestionar la salud de los ciudadanos de un día para otro y, menos todavía, no preocuparse por el problema. Es necesario plantearse a plazo las necesidades de una formación inicial, posteriormente continua, del cuerpo médico, la puesta en marcha de sistemas y de un espíritu de coordinación entre la medicina de ambulatorio y la medicina hospitalaria, la necesidad de acciones preventivas selectivas, el desarrollo de la epidemiología y de la estadística médica, la investigación, al igual que la difusión y la aplicación de los resultados; todo ello requiere tiempo, un tiempo de constante renovación. No nos podemos contentar simplemente con sacar partido de las enseñanzas pasadas o de los trabajos recientes, porque tanto unos como otros se superan constantemente o hay que ponerlos en cuestión.

Además, no hay que perder de vista que en una población con una fuerte proporción de personas de edad muy avanzada, un buen número de ellos son frágiles, en el sentido de que, aun cuando conserven su autonomía, ésta está amenazada por cualquier enfermedad que aceche un organismo que no tiene las facilidades de recuperación o de compensación de la edad adulta o de la juventud. De hecho, nuestras sociedades acumulan «supervivientes artificiales» con cierta facilidad, al precio de esfuerzos que no tienen siempre la eficacia deseable y que son cada vez más costosos.

Esto hace aparecer y refuerza un problema social que no ha sido aún resuelto mediante una respuesta satisfactoria; se trata de la "dependencia» que, independientemente de la edad, imposibilita o dificulta el desarrollo de la vida cotidiana. En el caso de los «dependientes mayores», las consecuencias son todavía de la dependencia y son más difíciles de superar debido a la avanzada edad; éste sería el caso cuando exige una compañía continua durante veinticuatro horas. Esta forma de ayuda, más social que médica, impone gastos suplementarios a la familia que se hace cargo o al Estado, que podría retractarse; de forma que el principio de un «subsidio dependiente» se ha convertido en un tema de actualidad en Francia, aunque encuentre dificultades en el Parlamento, más reticente a esta cuestión de lo que lo son las compañías de seguros y las mutuas.

Cuando las sociedades desarrolladas se preocupan por el aumento de los gastos de salud (que no son solamente gastos médicos), es lógico pensar que el envejecimiento de la población ayudará a controlar este proceso ¿No es más lógico temer que la evolución demográfica imponga opciones dolorosas, inclu- 
so trágicas, en ocasiones? En algún caso, que conocemos personalmente, no podemos dejar de pensar que, bajo buenos pretextos de gestión económica, un número creciente de pacientes ancianos pueden ser considerados como demasiado «caros» y, por esta razón, reenviados a sus hogares después de una grave intervención, sin que se hayan tomado todas las precauciones para asegurar una convalecencia eficaz. Puede ser que mañana estos ejemplos aislados se conviertan en la tónica, si no se toma conciencia acerca de las consecuencias que entraña el envejecimiento de la población, para la salud pública.

En este sentido habría que entender que sólo una pediatría eficaz permite o, más modestamente, facilita una vejez equilibrada. Una infancia enfermiza debilita las posibilidades de una trayectoria profesional plena y, por tanto, pone en cuestión la calidad de la jubilación y su duración. La unicidad del ser humano impone una medicina continua, lo que no quiere decir una medicina única. ¿De qué forma puede ser eficaz una geriatría si no sabe nada de los incidentes y, sobre todo, de los accidentes de la vida profesional? La dureza de las condiciones de trabajo y de las condiciones de vida imponen una vida corta, sin la fase intermedia hasta llegar a la auténtica vejez, que conocemos hoy en día y que no tiene precedente histórico. En este aspecto, el envejecimiento demográfico de tipo moderno supone una novedad.

Por otro lado, la salud pública no podrá progresar sin la participación de los interesados, es decir, sin una sensibilización por parte de la población de todas las edades. Múltiples estudios demuestran que el nivel de instrucción es el factor más discriminante en la mortalidad diferencial y, sin duda, de la morbilidad, pero no basta con mejorar la calidad de la educación en beneficio de los menos favorecidos, de los menos dotados; es necesario incluir, además, un espacio creciente dedicado a la educación sanitaria. Realmente, no habrá prevención eficaz sin una mejor preparación de la población afectada ${ }^{4}$.

\section{Las respuestas de la colectividad}

Ha llegado el momento de interrogarnos acerca de la naturaleza de las respuestas dadas por la comunidad y por el sesgo que introduce el Estado que la encarna, a las preguntas que plantea el envejecimiento de la población, tanto bajo su forma estática (grado), como bajo su forma dinámica (progresión).

Como lo demuestra la historia de este medio siglo, el Estado adopta frecuentemente leyes y reglamentos que tratan de compensar los efectos negativos del envejecimiento, limitándose a considerar el estrato más envejecido de la población, en relación a sus recursos mínimos, su vivienda o su salud. Este hecho se observa en toda Europa, independientemente de las diferencias políti-

${ }^{4}$ Estos argumentos están desarrollados y estructurados en el informe del Alto Comité de Población y Familia, "Vieillissement et Santé», publicado en Documentation Française, en 1992 (p. 107). 
cas y culturales. Algunos países, como Francia, más ambiciosos, intentan estructurar estas acciones, dándoles una cierta coherencia que se puede denominar "política de la vejez», título tomado de un famoso informe oficial publicado en 1962. Las diez personalidades, miembros de la Comisión, creada por Decreto de 8 de abril de 1960, y de un modo particular su presidente, el Sr. Pierre Laroque, tenían unas miras elevadas, y buen número de sus recomendaciones merecerían ser recordadas, entre otras cosas, porque no han surtido efecto. Actualmente, es más frecuente citar el Informe Laroque que presentar sus puntos fuertes, tales como la supresión de los límites de la edad «reglamentaria, convencional o habitual» (p. 264), o el rechazo de una "administración de la vejez que materializaría una segregación de las personas ancianas contra la cual no se sabía cómo luchar» (p. 275), o recordar, precisamente, esta condena a la segregación. Es precisamente a la Comisión a quien se debe el desarrollo de la ayuda doméstica, de la ayuda médica a domicilio y la idea de unos ingresos mínimos.

El contraste entre «decir» y «hacer» es aún más impactante, en relación con el VI Plan, dotado de un título prospectivo, "Envejecer mañana» ${ }^{5}$. La cuestión es si toda su insistencia para descartar el recurso de la edad, como criterio selectivo, ¿no ha sido suprimida por la legislación subsiguiente que ha reducido la edad de la jubilación presentando esta medida como un progreso social?

Adoptando una "política de la vejez», que no es, en resumidas cuentas, más que un catálogo no restrictivo de medidas, el Estado tiene el derecho a ciertas reivindicaciones justificadas por la mala situación de los ancianos desde la última guerra y conviene, a este respecto, hacer justicia a los poderes públicos desde entonces, pero, no obstante, queda por emprender una renovación en profundidad en este sentido.

En los textos propuestos o adoptados, raramente aparece la posibilidad de la intervención personal de los beneficiarios; por el contrario, parece que se esfuerzan en reducir su papel bajo el pretexto de permitirles "disfrutar de un descanso bien ganado». Esta "política» es, en efecto, concebida "para» y no "por». Logra tener una buena conciencia al prodigar ciertos apoyos; se desembaraza de los problemas espinosos y, en todo caso, evita suscitar ciertas preguntas embarazosas. Por ejemplo, parece que los «jóvenes jubilados» y los «mayores» disponen de una salud física y mental sin precedentes en la historia, pero entonces, ¿por qué se pone tanta firmeza en prohibirles toda participación activa, incluso a tiempo parcial, en la sociedad que ellos han contribuido a forjar?

Para merecer tal nombre, una "política de la vejez» debería defender, a cualquier precio, el mantenimiento de los ancianos en la sociedad, combatir las rigideces sociales y culturales que se oponen a ello y contribuir a una mayor valoración del potencial humano que poseen cientos de miles de ciu-

Comisariado General del Plan, grupo «Prospectiva de las personas mayores», «Vieillir demain», París, 1980, Documentation Française, p. 270. 
dadanos. ¿A quién puede servir una Secretaría de Estado para las personas ancianas, privada de toda competencia en los aspectos clave, jubilación, salud, empleo, y mezquinamente dotada? Una existencia en tales términos, ¿no reforzará, en el público, la opinión de que personas mayores tienen necesidad de ayuda y protección? De un modo más prosaico, los electores ancianos a los que miman los partidos son, en primer lugar, electores, dado que el envejecimiento de la población tiene, entre sus consecuencias, la modificación del cuerpo electoral.

¿Existen las personas ancianas en cuanto tales? Sí, si se las define en relación con una edad determinada, ¿pero determinada por quién y para qué? No son los demógrafos, ni los estadísticos del INSEE, quienes escogen o determinan la edad de la vejez, éstos no hacen más que utilizar la edad fijada por las leyes y los reglamentos para la jubilación. A pesar de ello, son muchos los que se oponen al descenso de la edad que convierte arbitrariamente en «viejos» a millones de "jóvenes jubilados». La elección de la edad es una decisión política ${ }^{6}$ y no una decisión científica; y además esta elección puede ser errónea. No lo serán si se tienen en cuenta ciertos handicaps, porque son las limitaciones, y no de la edad en sí misma, las que justifican una acción compensatoria. Tomemos un ejemplo simple: ¿Por qué sugerir una "política de vivienda para las personas mayores» cuando la gran mayoría tiene movilidad y agilidad? La dependencia que uno asocia tan fácilmente a la edad puede producirse después de un accidente o de una enfermedad invalidante; el sujeto, el "beneficiario» de la intervención, no es una persona anciana dependiente, sino una persona dependiente cuya avanzada edad no es una ventaja porque limita la eficacia de ciertas intervenciones (cirugía, prótesis, etc.) o justifica las contraindicaciones. Proporcionar una "tarjeta oro" para un jubilado, cuando los ingresos le han disminuido sensiblemente, es una medida socialmente justificada que mantiene a ese jubilado en la sociedad; concederla a toda persona que ha superado tal o cual edad, sólo tiene valor comercial. Nuestros contemporáneos mayores tienen algo mejor que aportarnos que su mera existencia.

De cara a una transformación profunda que provoque distorsiones de estructura mucho antes de la etapa de la jubilación, nuestras sociedades necesitan otro tipo de política, no una política de acompañamiento, de reparación, sino una política movilizadora y preventiva, que intervenga para dar impulso y no para prohibir a partir de la segunda mitad de la vida (si no antes), implicando de ese modo a las generaciones sucesivas. En la expresión «política del envejecimiento" (concepto lanzado y defendido por A. M. Guillemard), el término «envejecimiento» es revelador de un cierto dinamismo aplicado a una línea de acción intervencionista. Se abandona, pues, la concepción providencialista para entrar en la de la optimización de los recursos humanos, cualquiera que sea la edad.

${ }^{6}$ Cuando se adoptó, en su momento, los sesenta y cinco años, Bismarck estaba seguro de no poner en peligro los sistemas civiles de pensión. 
Desde esta óptica, debería entrar en juego una política del empleo que tenga en cuenta el envejecimiento interno de la población en edad acti$\mathrm{va}^{7}$. Tratar de revalorizar a las personas de cuarenta años y a sus predecesores que mañana serán mayoría, supone que la máquina económica no se agarrote; supone beneficiarse de la participación de los activos con experiencia, en lugar de inflar artificialmente a la población desempleada. De un modo más sutil, organizar una promoción estimulante supone movilizar a los más jóvenes (¿por qué mejorar su capacidad sin esperar recompensa?) y reforzar el potencial de la «segunda carrera». En estos momentos, la estructura de la pequeña explotación agrícola, el esqueleto del conjunto de la población agrícola, ofrece un ejemplo sorprendente. Con su estructura invertida (edad media de cincuenta y cinco años), el relevo generacional no tendrá lugar (los jóvenes no quieren aguardar a la marcha de los ancianos), hecho que producirá, en el futuro, un abultamiento brutal de los efectivos, que puede ser beneficioso en ciertas zonas de pequeña agricultura familiar polivalente, pero que será inquietante en otras donde triunfará la agricultura mecanizada a gran escala sin demasiado arraigo. El pequeño comercio tradicional del medio rural ha entrado en una fase aún más preocupante; carente de clientes, cede paso a las grandes superficies que frecuentan los jóvenes y, sin comercio, los pueblos mueren. Ayudando a su estructura demográfica será posible hablar de pueblos envejecidos, veillages.

\section{CONCLUSIONES. EL ENVEJECIMIENTO DEMOGRAFICO, UN DESAFIO A SUPERAR, UNA OPORTUNIDAD A PROVECHAR}

Las sociedades occidentales se encuentran en los albores del envejecimiento de sus poblaciones, en un mundo todavía marcado por un crecimiento demográfico excesivo. Decimos albores, porque la barrera del 20 por 100 de los ancianos de más de sesenta y cinco años no ha sido aún superada, si bien se anuncia para el inicio del próximo siglo.

Como ya se ha dicho, el envejecimiento se traducirá en una mutación profunda de la distribución por edades, imponiendo nuevas relaciones intergeneracionales. Si se acepta la perspectiva de una vida profesional que abarque algo menos de la mitad de la vida y que ocupe a un tercio de la población, habrá que aceptar una profunda redefinición de nuestros sistemas socioeconómicos que no se beneficiarán más de un seccionamiento de la vida y de la población en etapas autónomas (escuela-trabajo-jubilación) y que, por el contrario, reclaman de cada uno lo mejor de sí mismos y de su talento. Lo que parece claro es la necesidad de una optimización de los recursos humanos, especialmente en el seno de la familia. ¿Por qué ayudar a los que se ocupan de sus hijos y negar

7 En Francia, la relación 40-59 años/20-39 será positiva entre el 2000-2005; en la Europa de los Doce llegará a serlo, probablemente, más pronto. 
esta ayuda a los que se ocupan de sus padres ancianos dependientes? Esta optimización sería el contrapeso las medidas que fraccionan la vida y la sociedad en secciones estancadas al capricho de los actores políticos. ¿Cómo conciliar este seccionamiento con la preocupación por «la solidaridad necesaria del conjunto de la población y el respeto por las personas de edad avanzada»? (Informe Laroque, p. 274).

En la medida en que la fuerza manual sea menos necesaria que en momentos precedentes, gracias a que su relevo queda asegurado por la mecanización, la electrónica y la informática, nuestras sociedades se verán menos penalizadas por su envejecimiento y podrán dedicar más energía a las actividades de servicio ejercidas según las disponibilidades de cada uno, sin criterios de edad o sexo.

Con vistas al desafío a superar y a la oportunidad a aprovechar, será preciso que el envejecimiento psico-sociológico no se oponga a las transformaciones necesarias. Es aquí donde la responsabilidad de los poderes públicos es inmensa, no podrán contentarse con seguir la corriente. A fin de poder convencer, tendrán que estar, en primer lugar, íntimamente convencidos de la importancia y de la urgencia de su tarea frente a un fenómeno que perturba porque trastorna los esquemas y las referencias heredadas de un tiempo en el que las poblaciones no tenían ni la misma estructura ni la misma composición. A partir de ahora, las áreas sobreenvejecidas hacen imperativas intervenciones multiformes que podrán ayudar a formular soluciones aplicables a nivel nacional y europeo. También en este caso el desafío lanzado conlleva una oportunidad a aprovechar. En este aspecto, los demógrafos pueden y deben contribuir a revalorizar el carácter potencialmente rico de un fenómeno que no tiene porqué limitarse a los aspectos negativos.

\section{RESUMEN}

El envejecimiento de las poblaciones europeas es un fenómeno nuevo e imparable. Precisamente por su novedad plantea problemas que estas sociedades no están preparadas para resolver de forma satisfactoria. En su resolución habrá que tener en cuenta toda una serie de factores, entre los que cabe destacar las políticas sociales, en especial las políticas de familia y las políticas de salud, sin olvidar la importancia crucial de la solidaridad intergeneracional.

\section{ABSTRACT}

The ageing of the European population is a new, unstoppable phenomenon. This newness raises problems that European society is as yet unable to resolve satisfactorily. In order to address them, a number of factors have to be taken into account, notably social policies - especially family-oriented and health-care policies — without losing sight of the crucial importance of intergenerational solidarity. 